\title{
Aggregation of Chameleon Peptides: Implications of $\alpha$-Helicity in Fibril Formation
}

Bongkeun Kim ${ }^{\dagger \neq}$, Thanh D. Do ${ }^{\ddagger}$, Eric Y. Hayden ${ }^{\dagger}$, David B. Teplow ${ }^{\dagger}$, Michael T. Bowers ${ }^{\ddagger}$, JoanEmma Shea ${ }^{\ddagger \neq, *}$

${ }^{\dagger}$ Materials Research Laboratory, ${ }^{\ddagger}$ Department of Chemistry and Biochemistry, ${ }^{\ddagger}$ Department of Physics, University of California, Santa Barbara, CA 93106-9510

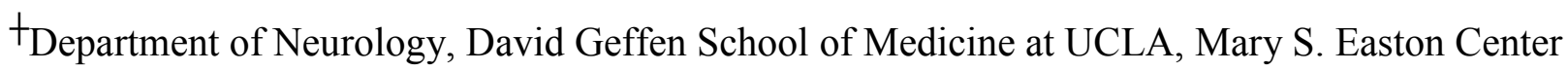
for Alzheimer's Disease Research at UCLA, and Brain Research Institute and Molecular Biology Institute, University of California, 635 Charles Young Drive South, Los Angeles, California 90095, United States

${ }^{\gamma}$ Current address: Department of Chemistry and the Beckman Institute, University of Illinois at Urbana-Champaign, Urbana, Illinois 61801, United States

To whom correspondence should be addressed. Email: *shea@chem.ucsb.edu 


\section{S1. Materials and Methods}

\section{S1.1 Chamelon-HE sequence}

The 16-residue long HE- $\alpha$ (residue id: 90-105 of PDB 1N81 ${ }^{1}$ ) and HE- $\beta$ (residue id: 224-239 of PDB $1 Q^{2} L^{2}$ ) sequences are peptides that contain the HE chameleon sequence and adopt $\alpha$-helix and $\beta$-sheet conformations in the proteins, respectively. The HE- $\alpha$ and HE- $\beta$ peptides were capped with acetylated N-terminal (ACE) and N-methyl amidated C-terminal (NME) protecting groups to minimize the possibilities of trappings by the charged ending groups which might prevent equilibrations of formed secondary structures.

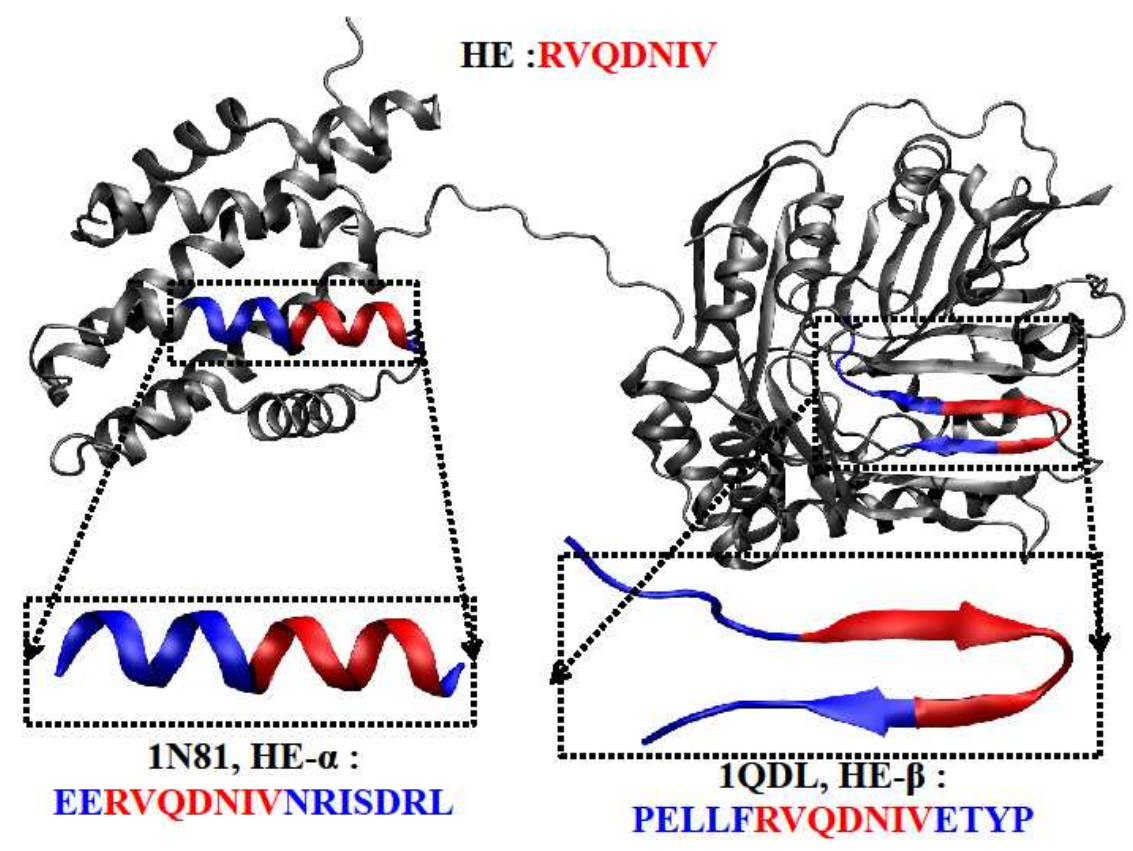

Figure S1. A chameleon-HE sequence RVQDNIV (red) in 1N81 ( $\alpha$-helix) and in 1QDL ( $\beta$ sheet). The sequence fragments taken by the dotted boxes, HE- $\alpha$ (helix-sheet sequence forms $\alpha$ helix conformation) and HE- $\beta$ (helix-sheet sequence forms $\beta$-sheet/hairpin) are isolated from the original PDB structure and high-lighted in blue. The isolated HE- $\beta$ sequence does not have the 
same number of the $\beta$-sheet residues as in the original PDB structure because of the absence of complementary neighboring $\beta$-strands. The figure was prepared by VMD. ${ }^{3}$

\section{S1.1 Replica Exchange Molecular Dynamics.}

\section{Theoretical setup and methods.}

Implicit solvent T-REMD. We used the temperature based REMD simulations with the AMBER 11 simulation suite. ${ }^{4-5}$ All six peptides, HS, HE, HS- $\alpha$, HS- $\beta$, HE- $\alpha$ and HE- $\beta$ were modeled using the AMBER all-atom point-charge protein force field, AMBER ff96 which is known to be a fairly unbiased force-field for both $\alpha$ and $\beta$ secondary structure prediction when combined with a generalized-Born implicit solvent model (igb $=5) \cdot{ }^{6}$ Recent publications ${ }^{7-8}$ have shown that this combination worked for prediction of reasonable structures for $\alpha$ protein, $\beta$ protein and $\alpha / \beta$ proteins. All REMD simulations were performed with an effective salt concentration of $0.1 \mathrm{M}$. In each peptide fragment simulation, 1,000 steps of an initial minimization were performed to get the input structure for replicas, and 50-ps equilibration at target temperatures for all replicas were run to get initial replicas for production REMD stage. To maintain approximately $30 \%$ of successful exchange rate, we chose temperature ranges and the number of replicas for each peptide by using the optimized algorithm. ${ }^{9}$ Eight replicas were set up with exponentially spaced targeting temperatures from 280 to $500 \mathrm{~K}(286.2,309.1,333.8,360.5$, 389.3, 420.4, 454.0, 490.3) for small chameleon-HS and HE peptides and ten replicas were set up with targeting temperatures from 280 to $500 \mathrm{~K}(287.4,306.0,325.7,346.7,369.0,392.8$, $418.2,445.2,473.9,504.4)$ for 16 residues long HE- $\alpha$ and HE- $\beta$ peptides and finally 12 replicas were set up with targeting temperatures from 280 to $500 \mathrm{~K}$ (283.8, 298.4, 313.7, 329.8, 346.7, $364.5,383.1,402.8,423.4,445.2,468.0)$ for 24 residues long HS- $\alpha$ and HS- $\beta$ peptides. The time 
step was set to $2.0 \mathrm{fs}$ and the exchange frequency between neighboring replicas was set to every 1,000 steps and coordinates were also saved in this period. The temperature was regulated by Langevin thermostat ${ }^{10}$ with the collision frequency $\gamma=1.0 \mathrm{ps}-1$ and to constrain bonds involving hydrogen, $\mathrm{SHAKE}^{11}$ was turned on. The total exchange attempts were set to 75,000 for HE, HS, HE- $\beta$ and HE- $\alpha$ and 100,000 for HS- $\alpha$ and HS- $\beta$, so simulations were executed for 150 ns for HE family REMD set and 250 ns for HS- $\alpha$ and HS- $\beta$.

In vacuum simulation. The structures with highest number of the secondary structures from HS- $\alpha$ and HS- $\beta$ were used as an input structures of REMD simulations in vacuum to make comparisons of theoretical cross section with IM-MS experiment. All settings for REMD simulations in vacuum are exactly same as for the previous solution phases except turning off the generalized Born model (igb=0). The simulations were executed for $150 \mathrm{~ns}$ and the trajectories of the last $50 \mathrm{~ns}$ were used to analyze the data.

Analysis Methods. All the secondary structures were assigned to the amino acids of PDB snapshots converted from AMBER trajectories by the DSSP program. ${ }^{12}$ We took the average populations of all different secondary structures in every 25 ns to determine whether RMSD simulations were converged (see Supporting Information, S1). According to the relatively constant behaviors of the secondary structure populations between $100 \mathrm{~ns}$ to $150 \mathrm{~ns}$ time intervals for HE- $\beta$ and HE- $\alpha$ and between 200 ns to 250 ns time intervals for HS- $\alpha$ and HS- $\beta$, we chose the last $50 \mathrm{~ns}$ of the time frames to do further analyses. The isolated chameleon sequences, HE and HS, did not show the constant behaviors of the secondary structure populations in any time interval, so we used 100 ns to $150 \mathrm{~ns}$ time intervals for consistency. Free energy landscapes for folding of chameleon-HS and chameleon-HE peptides were characterized by plotting potential of mean force (PMF) as a function of root mean square deviations (RMSD) to the 
reference $\alpha$-helix PDB fragments (1JIG for HS and 1N81 for HE) and RMSDs to the reference $\beta$ PDB fragments (1P35 for HS and 1QDL for HE) using the backbone atoms, N, C $\alpha$ and $\mathrm{C}$ at 309.1 K. To draw free energy landscapes for folding of chameleon- HS- $\alpha$ and HS- $\beta$, and chameleon- HE- $\alpha$ and HE- $\beta$ peptides, potential of mean force (PMF) was plotted as a function of the radius of gyration, Rg and RMSD to the reference PDB structure for each peptide. The backbone atoms, $\mathrm{N}, \mathrm{C} \alpha$ and $\mathrm{C}$ were used to calculate RMSDs and only residues which are assigned to $\alpha$-helix or $\beta$-strand/sheet/hairpin secondary structures were counted as an input for RMSD calculations because once the reference peptides are fragmented from whole proteins in the PDB structures, they miss a part of secondary structures because of the absence of their correspondent secondary structure especially $\beta$-strand and $\beta$-sheet. So we used backbone atoms from 3-16 residues ( $\alpha$-helix region) of the reference fragment from PDB id $=1$ N81 for HE- $\alpha$ peptide and backbone atoms from 6-13 residues ( $\beta$-sheet/hairpin region) of the reference fragment from PDB id = 1QDL for HE- $\beta$ peptide. Again we used backbone atoms from 2-23 residues ( $\alpha$-helix region) of the reference fragment from PDB id $=1 \mathrm{JIG}$ for HS- $\alpha$ peptide and backbone atoms from 6-19 residues (two $\beta$-strand region) of the reference fragment from PDB id $=1 \mathrm{P} 35$ for HS $-\beta$ peptide. PMF curves were calculated at the closest temperature to $310 \mathrm{~K}$ of the given temperatures in REMD simulations, $309.1 \mathrm{~K}$ for chameleon-HS and HE peptides, $306.0 \mathrm{~K}$ for HE- $\alpha$ and HE- $\beta$ peptides and $313.7 \mathrm{~K}$ for HS- $\alpha$ and HS- $\beta$ peptides and Weighted Histogram Analysis Methods (WHAM) ${ }^{13-15}$ was used to calculate PMFs. Data from all temperatures were taken into account during PMF calculations. Clustering analysis using MMTSB tool set $^{16}$ was applied to detect most populated conformations at the same temperature with each PMF calculation, in which the structure similarity metric was based on the pair-wise RMSD over backbone $\mathrm{N}, \mathrm{C} \alpha, \mathrm{C}$ atoms of the peptides and the RMSD cutoff was set to $3.0 \AA$ to capture major 
structural features. The areas of centroid structures were pointed on the free energy landscapes if they were within the first 5 th rank or about more than $5 \%$ of population. The lowest RMSD trajectory snapshot from each counted centroid that has the large number of neighboring structures within the cutoff, was selected as the representative structure of the centroid family and drawn on its PMF landscape. The total number of snapshots within the family as calculated to population percentage over the total ensemble indicates the stability of the structure of peptides. Theoretical collision cross sections were obtained as an average of 50 structures per cluster. In order to test the effect from structural properties, 2 clusters in each chameleon sequence with contexts were chosen by small and large radius of gyration $(\mathrm{Rg})$.

\section{S1.2 Ion-mobility mass spectrometry.}

Peptides and sample preparation. HS- $\beta$ (KIFCNGFIYDKKSKVLYVKLHNVT, free termini) was synthesized by FMOC ( $N$-(9-fluorenyl)methoxycarbonyl) chemistry. The peptides were purified by reverse-phase HPLC and characterized by mass spectrometry and amino acid analysis. HS- $\alpha$ (Ac-VEVLNKQVANWNVLYVKLHNYHWY-NHE, acetylated N-terminus and N-methylated C-terminus, >95\%) was purchased from Genscript (Piscataway, NJ, USA) and used without further purification. Stock samples were prepared at the concentration of $1 \mathrm{mg} / \mathrm{mL}$ in hexafluoroisopropanol (TCI America, USA) to disrupt oligomer formation and prevent aggregation. Aliquots of stock samples were evaporated before solvents were added to dilute the spray samples to the final concentration of $150 \mu \mathrm{M}$. Water (Water, HPLC grade, $\mathrm{pH}=6.5$ ) and ammonium acetate buffer $(\mathrm{pH}=7.0)$ were used as solvents.

Methods. In these experiments, ions were generated through means of nano-electrospray ionization (nano-ESI) and stored in a source ion funnel and subsequently pulsed into a solventfree drift cell filled with He gas at high pressure. Several factors can affect the extent of analyte 
charges in ESI including the molecular conformations, solvent tension, gas-phase basicity, denaturing agents, instrumental parameters, etc. ${ }^{17-19}$ Moreover, analyte ions produced from n-ESI have observed charge states typically not the same as those in bulk solution. n-ESI can produce gas-phase ions with opposite charges to that in solution. ${ }^{17,}{ }^{19}$ For example, our group has shown that ESI of $A \beta_{42}$ peptide under the same experimental condition produces both positive and negative charge ions while the native state of $\mathrm{A} \beta_{42}$ in solution has $z=-3$ charge state..$^{20-21}$ Another example is octaethylporphyrin has predominantly $z=+1$ charge state in gas-phase while doubly protonated species is native in solution. ${ }^{22}$ However, the difference (or similarity) in charge states between gas-phase ions and native solution structures does not necessarily rule out the possibility of obtaining dehydrated solution-phase structures whose secondary structure is retained. A distribution of charge states is often observed, where the range of the distribution depends on the size of the system. Thus, we expect systems with similar sizes to have similar charge state distributions under the same experimental conditions. The charge states closer to the native charge states often give structures with cross sections similar to those of the gas-phase, whereas high charge-states often stabilize denaturing conformations, which make the charge states in the middle range the best candidates to probe for native structures. ${ }^{21,23}$ In the context of this paper, we compare our experimental result with the most abundant charge states for all systems. IM-MS offers a direct method to probe the three dimensional structures of molecules by measuring an average collision cross section of a mass-selected ion corresponding to the species of interest. Ions drift through the cell under the influence of a weak electrical field. During the drifting process collisions between the ions and He buffer gas molecules occur, which create a frictional force countering the force gained by the field. Ions were separated on the basis of their sizes, shapes and charges which impacted both their travel velocities and their mass to charge 
ratios. The arrival time distributions (ATDs) of mass-selected ions were measured as a function of the pressure to drift voltage ratios, and used to determine the reduced mobility $K_{0}$ and experimental collision cross sections $\sigma$ as shown in Equation $1 .^{24}$

$\sigma \approx \Omega_{\text {avg }}=\frac{(18 \pi)^{1 / 2}}{16}\left[\frac{1}{m}+\frac{1}{m_{b}}\right]^{1 / 2} \frac{z e}{\left(k_{B} T\right)^{1 / 2}} \frac{1}{K_{0}} \frac{1}{N}$ (Eq. 1)

of the ion, $N$ is the buffer gas density and $\Omega_{\text {avg }}$ is the average collision cross section integral, which approximates to be the same as the average collision cross section $\sigma$.

In IM-MS experiment, the secondary structure of the system plays a critical role in determining whether solution-phase structure can survive. In general, unstructured (random coil) peptides are likely to collapse into gas-phase compact structures when being introduced into the gas phase. On the other hand, well-defined conformations such as $\alpha$-helix or $\beta$-hairpin are easily preserved. A well-known example is $\mathrm{Ala}_{n} \mathrm{LysH}^{+}(n>7)$ adopting stable helix conformations in both solution and vacuum. ${ }^{25}$

The flux of ions exiting the drift cell can be calculated theoretically assumed that the ion packet takes the form of a periodic delta function. Based on this information, the experimental ATDs can be fitted with different features corresponding to different species having the same $\mathrm{m} / \mathrm{z}$ value, following Equation 2. ${ }^{26}$

$\theta(0, z, t)=\frac{s \cdot a \cdot e^{-\alpha t}}{4\left(\pi D_{L} t\right)^{1 / 2}}\left(v_{d}+\frac{z}{t}\right)\left[1-e^{\left(\left(-r_{0}^{2} / 4 D_{T} t\right)\right.}\right] e^{\left(-\left(z-v_{d} t\right)^{2} / 4 D_{L} t\right)}$ (Eq. 2)

where $s$ is the initial ion density, $a$ is the area of the exit aperture, $\alpha$ is the loss of ions due to reactions inside the drift cell, $D_{T}$ and $D_{L}$ are transverse and longitudinal diffusion coefficients, $z$ is the distance the ions travel and $r_{0}$ is the radius of the initial ion packet. 


\section{S1.3 Transmission Electron Microscopy (TEM)}

Eight $\mu \mathrm{L}$ of peptide at $\mathrm{t}=0$ or $\mathrm{t}=480$ hours, was spotted onto a carbon-coated Formvar grid (Electron Microscopy Sciences, Hatfield, PA) and incubated for 2 min. The droplet then was displaced with an equal volume of $1 \%(\mathrm{w} / \mathrm{v})$ filtered $(0.2 \mu \mathrm{M})$ uranyl acetate in water (Electron Microscopy Sciences). This solution was wicked off and then the grid was air-dried. Samples were examined in an operator-blinded fashion, using a JEOL 1200 EX transmission electron microscope with an accelerating voltage of $80 \mathrm{kV}$. Digital images were analyzed with ImageJ $1.43 r$.

S1.4 Circular dichroism (CD). The circular dichroism spectra of HS- $\beta$ and HS- $\alpha$ in water and ammonium acetate buffer $(\mathrm{pH}=7.0)$ at the concentration of $0.5-1.0 \mathrm{mg} / \mathrm{mL}$ were obtained using an Aviv circular dichroism spectrometer model 202 (Instruments Inc.) in a $0.1 \mathrm{~cm}$ cuvette. The scan range was $260-185 \mathrm{~nm}$ with $1.00-\mathrm{nm}$ step and an average time per step of 5 seconds. Each spectrum was an average of two consecutive scans and subtracted by either water or ammonium acetate blank spectra and reproduced without further smoothing. Time-course CD spectra of peptides was acquired immediately after sample preparation or following $\mathrm{t}=1,2,3$, 23, 48, 72, 120, 168, 216, 360 and 480 hours on a J-810 spectrapolarimeter (JASCO, Tokyo, Japan). Select time points are shown for clear presentation. $150 \mu \mathrm{M}$ peptide was incubated in 1 mm path length quartz cuvettes (Hellma, Forest Hills, NY) at $22^{\circ} \mathrm{C}$ without agitation. Spectra were recorded at $22^{\circ} \mathrm{C}$ from $195-260 \mathrm{~nm}$ at $0.2 \mathrm{~nm}$ resolution with a scan rate of $100 \mathrm{~nm} / \mathrm{min}$. Ten scans were acquired and averaged for each sample. 


\section{S2. REMD simulations}

\section{S2.1 Convergence of REMD simulations}
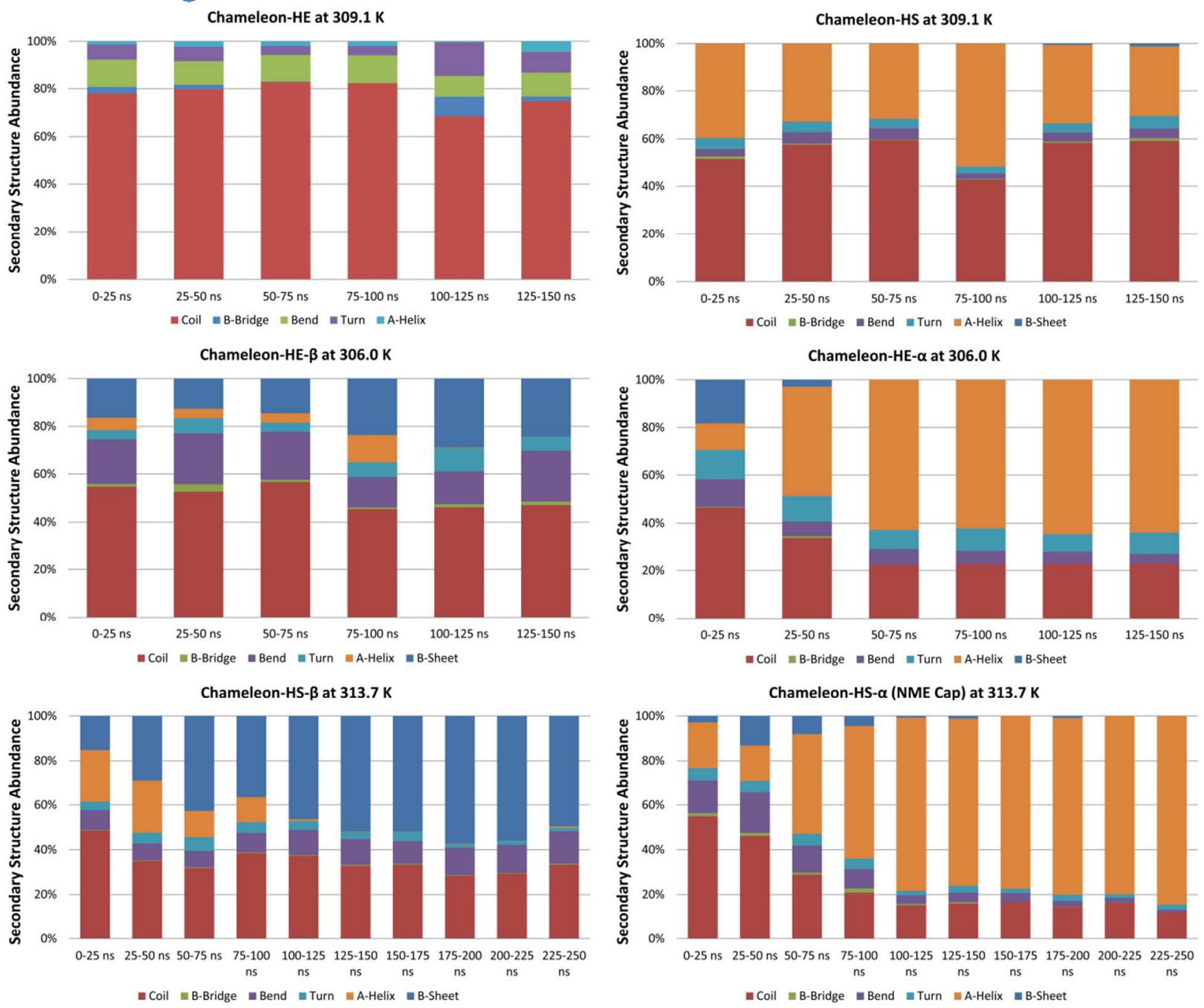

Figure S2. A typical example showing the convergence of REMD simulations in implicit water of chameleon sequences and their derivative is depicted. The total up to $250 \mathrm{~ns}$ sampling of all 6 sequences in the implicit solvent environment $(\mathrm{igb}=5)$ are equally divided into $25 \mathrm{~ns}$ time period for secondary structure abundance.

S2.2 PMF landscapes of chameleon-HE and HE- $\alpha$ and HE- $\beta$ sequences 

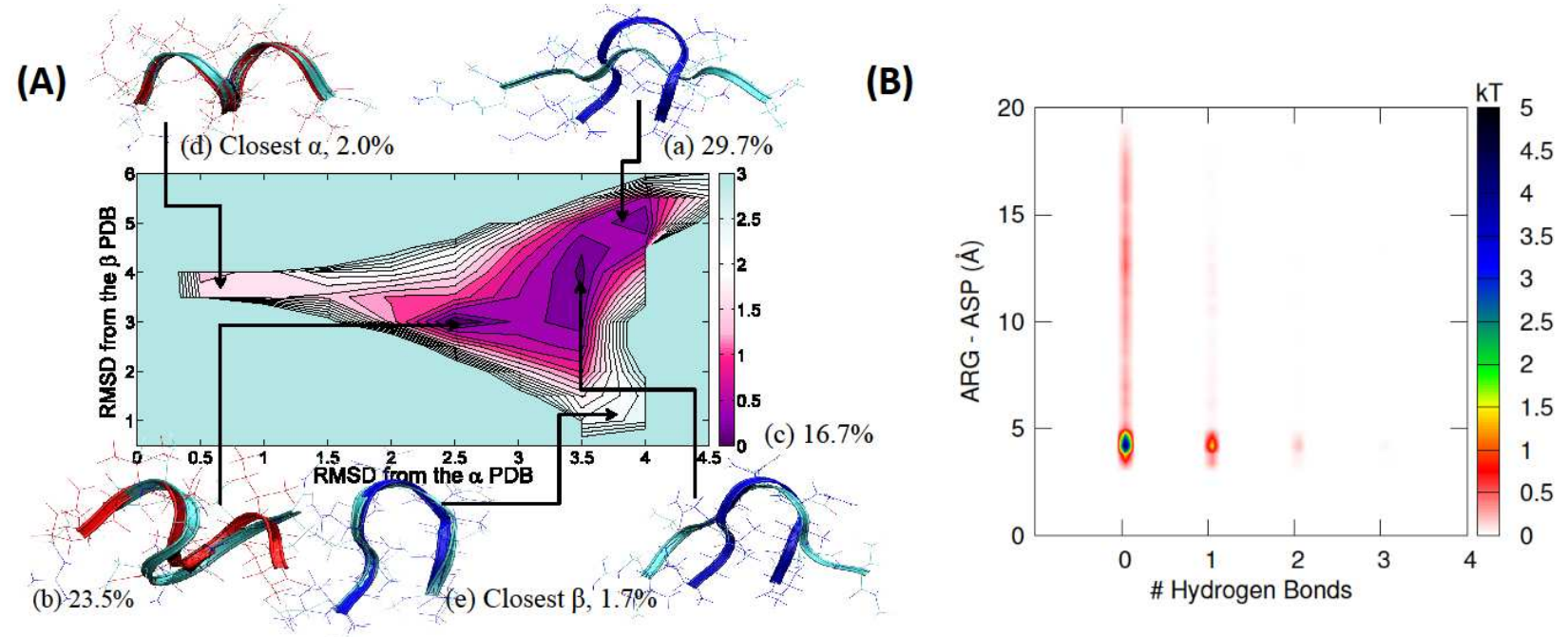

Figure S3. (A) PMF landscape of the chameleon-HE sequence fragment along with the result of cluster analysis. X-axis of the PMF graph is the RMSD from the reference $\alpha$-helix fragment of the protein, PDB id $=1 \mathrm{~N} 81$ and $\mathrm{Y}$-axis of the PMF graph is the RMSD from the reference $\beta$ strand fragment of the protein, PDB id = 1QDL. Each of (a), (b), and (c) structures was taken by choosing the lowest RMSD structure from each centroid and ordered by the populations indicated above. (d) and (e) structures are indicated to show the two closest structures' populations of $\alpha$-helix and $\beta$-sheet/hairpin reference structures. The red ribbon structure indicates the reference $\alpha$-helix structure and the blue ribbon structure does the reference $\beta$-strand structure. The energy unit on this PMF landscape is kcal/mol. (C) Population densities of monomeric salt bridges indicated by the relative proximities between arginine and aspartic acid and forming intramolecular hydrogen bonds are plotted for chameleon-HE sequence fragment. 


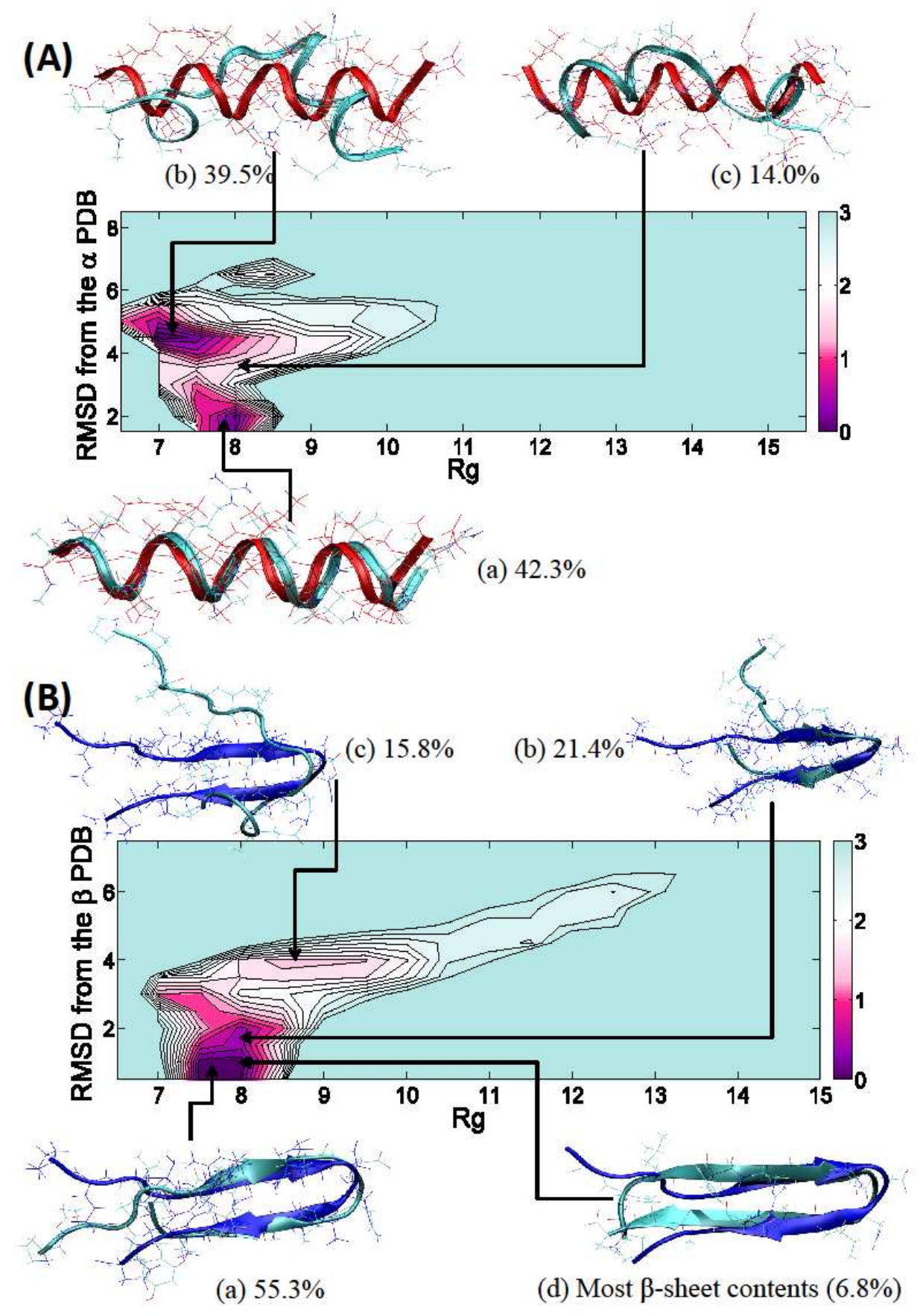

Figure S4. (A) PMF landscape of the chameleon-HE- $\alpha$ sequence fragment along with the result of cluster analysis. $\mathrm{X}$-axis of the PMF graph is the radius of gyration, $\mathrm{Rg}$ and $\mathrm{Y}$-axis of the PMF graph is the RMSD from the reference $\alpha$-helix fragment of the protein, PDB id $=1 \mathrm{~N} 81$. Each of (a), (b), and (c) structures was taken by choosing the lowest RMSD structure from each centroid 
and ordered by the populations indicated above. The red ribbon structure indicates the reference $\alpha$-helix structure. (B) PMF landscape of the chameleon-HE- $\beta$ sequence fragment along with the result of cluster analysis. X-axis of the PMF graph is the radius of gyration, Rg and Y-axis of the PMF graph is the RMSD from the reference $\beta$-sheet/hairpin fragment of the protein, PDB id $=$ 1QDL. Each of (a), (b), and (c) structures was taken by choosing the lowest RMSD structure from each centroid and ordered by the populations indicated above. The structure (d) populate by $6.8 \%$ was depicted to show the snapshot having the most $\beta$-sheet contents. The blue ribbon structure indicates the reference $\beta$-sheet/hairpin structure. All ribbon structures were prepared using VMD. ${ }^{27}$ The energy unit on this PMF landscape is kcal $/ \mathrm{mol}$.

HE- $\boldsymbol{\alpha}$. According to the PMF landscape depicted in Figure S4A by using the last $50 \mathrm{~ns}$ of total 150 ns REMD simulation, the most popular centroid occupied $42.3 \%$ and its helical contents calculated by the DSSP program ${ }^{12}$ were ranged from 12 to $14(49.9 \%$ of 12 and $50.1 \%$ of 14$)$ and the reference structure also had 14 helical contents. The structure comparison was depicted on Supporting Information Figure S4A centroid (a). The second most popular centroid had 39.5\% of population and about $8 \sim 12$ helical contents (see Figure S4A centroid (b)) and those helical contents were located from the N-terminus. And the third popular centroid had $14.0 \%$ of population and it was filled with the centroid which had $4 \sim 6$ helical contents with no sign of $\beta$ sheet contents and the helix began to fold into $\alpha$-helix from the C-terminus (see Figure S4A centroid (c)). The lowest RMSD from the reference $\alpha$-helix PDB structure to the structures in centroid (a) in Figure S4A is $1.65 \AA$.

HE- $\boldsymbol{\beta}$. The most populated structure depicted on Figure S4B centroid (a) calculated by cluster analysis had $55.3 \%$ population and its $\beta$-sheet contents calculated by the DSSP program ${ }^{12}$ were ranged from 8 to 14 including $\beta$-turn (the reference structure also had $8 \beta$-contents including 
hairpin structures). The second most popular centroid had $21.4 \%$ of population and about $6 \sim 8$ $\beta$-contents and the closest RMSD snapshot from the centroid was depicted on Figure S4B centroid (b). The third centroid was populated by $15.8 \%$ (Figure S4B (c)) and it had no $\beta$ contents. In the centroid (a) area, especially the snapshot for the most $\beta$-contents, 14 including $\beta$ sheet and hairpin were depicted as Figure S4B centroid (d) and its population was $6.8 \%$. In the last $50 \mathrm{~ns}$ of simulation, only a small observation, $0.4 \%$ was made by the structures which had any helical contents along with $\beta$-sheet/hairpin contents and those helical contents were in between 4 to 8 . The lowest RMSD from the reference $\beta$-sheet/hairpin PDB structure to the structures in the region of Figure S4B centroid (a) is $0.36 \AA$. The small RMSD result was because we took 6 to 13 residues from the N-terminus to calculate the RMSDs to avoid misalignment due to the residues from both $\mathrm{N}$ - and C-terminals where they are interacting with other $\beta$-strand in the original PDB structure.

These point mutations also induced $\alpha$-helix sequence (HE- $\alpha, 95.8 \% \alpha$-helix) to form $\beta$-sheet favored structures (HE- $\alpha \rightarrow \beta, 44.5 \% \beta$-sheet) as depicted on Figure S5A and $\beta$-sheet sequence (HE- $\beta, 83.5 \% \beta$-sheet) to form $\alpha$-helix favored structures (HE- $\beta \rightarrow \alpha, 77.6 \% \alpha$-helix) as also shown on Figure S5B. 


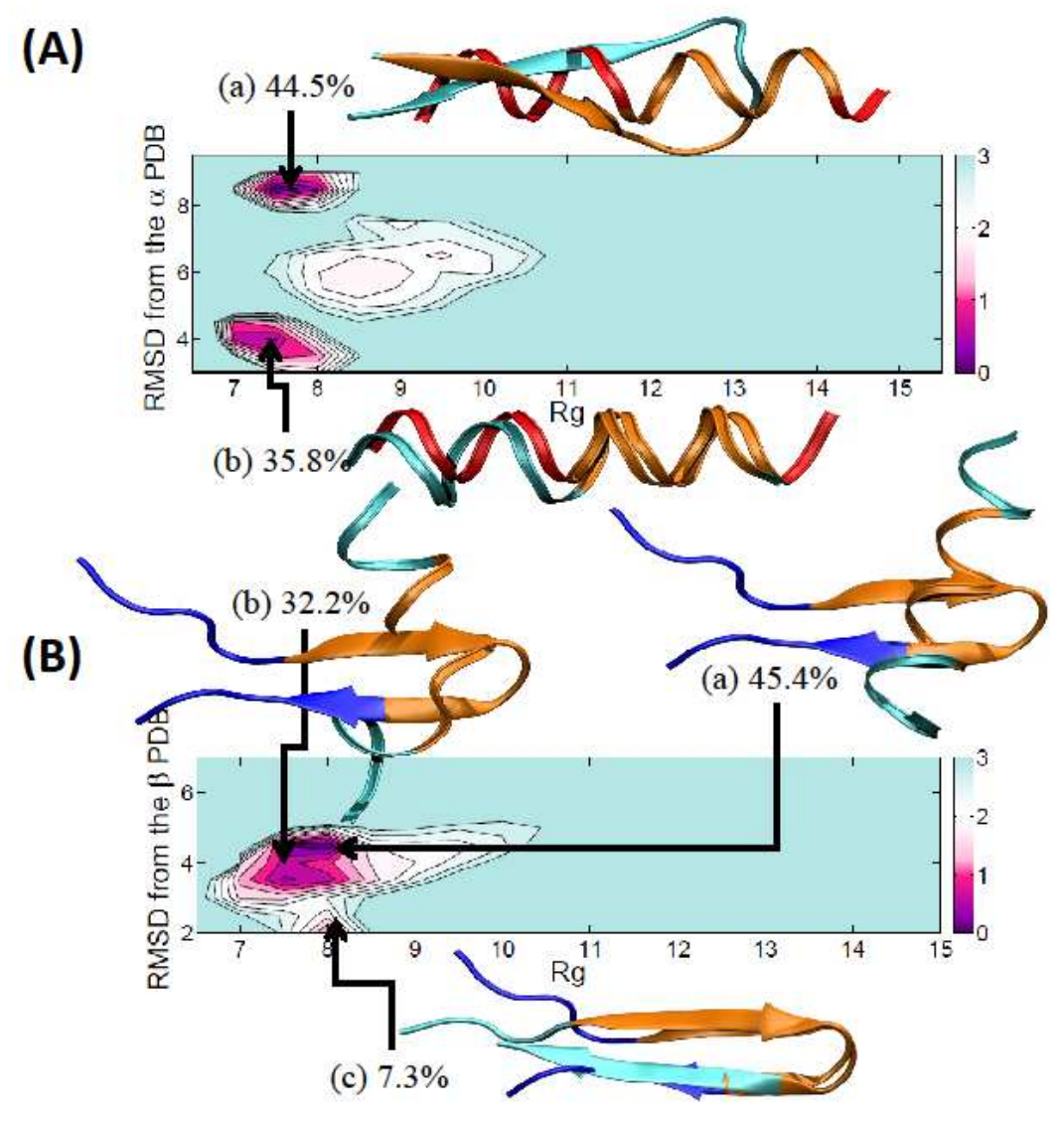

Figure S5. (A) PMF landscape of the chameleon-HE- $\alpha \rightarrow \beta$ sequence fragment (VVRVQDNIVVRISVRL, cyan: mutation, red: chameleon) along with the result of cluster analysis. $\mathrm{X}$-axis of the PMF graph is the radius of gyration, $\mathrm{Rg}$ and $\mathrm{Y}$-axis of the PMF graph is the RMSD from the reference $\alpha$-helix fragment of the protein, PDB id $=1$ N81. Each of (a) and (b) structures was taken by choosing the highest populations indicated above. The red ribbon structure indicates the reference $\alpha$-helix structure. (B) PMF landscape of the chameleon-HE$\beta \rightarrow \alpha$ sequence fragment (PELLLRVQDNIVELLP, cyan: mutation, red: chameleon) along with the result of cluster analysis. $\mathrm{X}$-axis of the PMF graph is the radius of gyration, Rg and Y-axis of the PMF graph is the RMSD from the reference $\beta$-sheet/hairpin fragment of the protein, PDB id $=1 \mathrm{QDL}$. Each of (a), (b), and (c) structures was taken by choosing the highest populations 
indicated above. The blue ribbon structure indicates the reference $\beta$-sheet/hairpin structure. Gold ribbons depict the common chameleon sequence. All ribbon structures were prepared using VMD. ${ }^{3}$ The energy unit on this PMF landscape is $\mathrm{kcal} / \mathrm{mol}$.

\section{S3. Circular Dichroism}

Circular dichroism probes the secondary structures of the proteins mainly through intense signals in the regions of 215-230 nm and 185-200 nm. The former one corresponds to electrically prohibited but magnetically permitted $\mathrm{n} \rightarrow \pi^{*}$, which often appears as a negative band at $222 \mathrm{~nm}$ ( $\alpha$-helix) or a negative band at $215-218 \mathrm{~nm}$ ( $\beta$-sheet). The latter one corresponds to $\pi_{0} \rightarrow \pi$ transition a positive band at $190 \mathrm{~nm}$ and a negative band at $208 \mathrm{~nm}$ ( $\alpha$-helix), or a positive band at $198 \mathrm{~nm}$ ( $\beta$-sheet). In addition, a negative peak in the $185-200 \mathrm{~nm}$ is often responsible for random coil structures. ${ }^{28}$ The presence of different conformations in bulk solution can give rise to variations. Here in our far-UV spectra in Figure S9, the HS- $\beta$ sample shows a clear negative peak in 200-230 nm region with a positive band at $185-200 \mathrm{~nm}$ region, which implies that $\beta$-hairpin is the dominant species, as predicted by molecular dynamics simulations. No significant difference is observed between the samples in water and acetate buffer, although the negative peak shifts slightly to the high frequency region for the water sample. The HS- $\alpha$ sample shows two clear negative bands at 200$215 \mathrm{~nm}\left(\pi_{0} \rightarrow \pi\right)$ and $215-230 \mathrm{~nm}\left(\mathrm{n} \rightarrow \pi^{*}\right)$. The positive band at 198 is not as intense as

expected for a $\alpha$-helix structure, which can be due to several factors including the sensitivity of our instrument in this region, and the presence of small intermediate oligomers whose structures may not be completely helical. But the overall shape of the spectra suggests the dominant species to be $\alpha$-helix. 

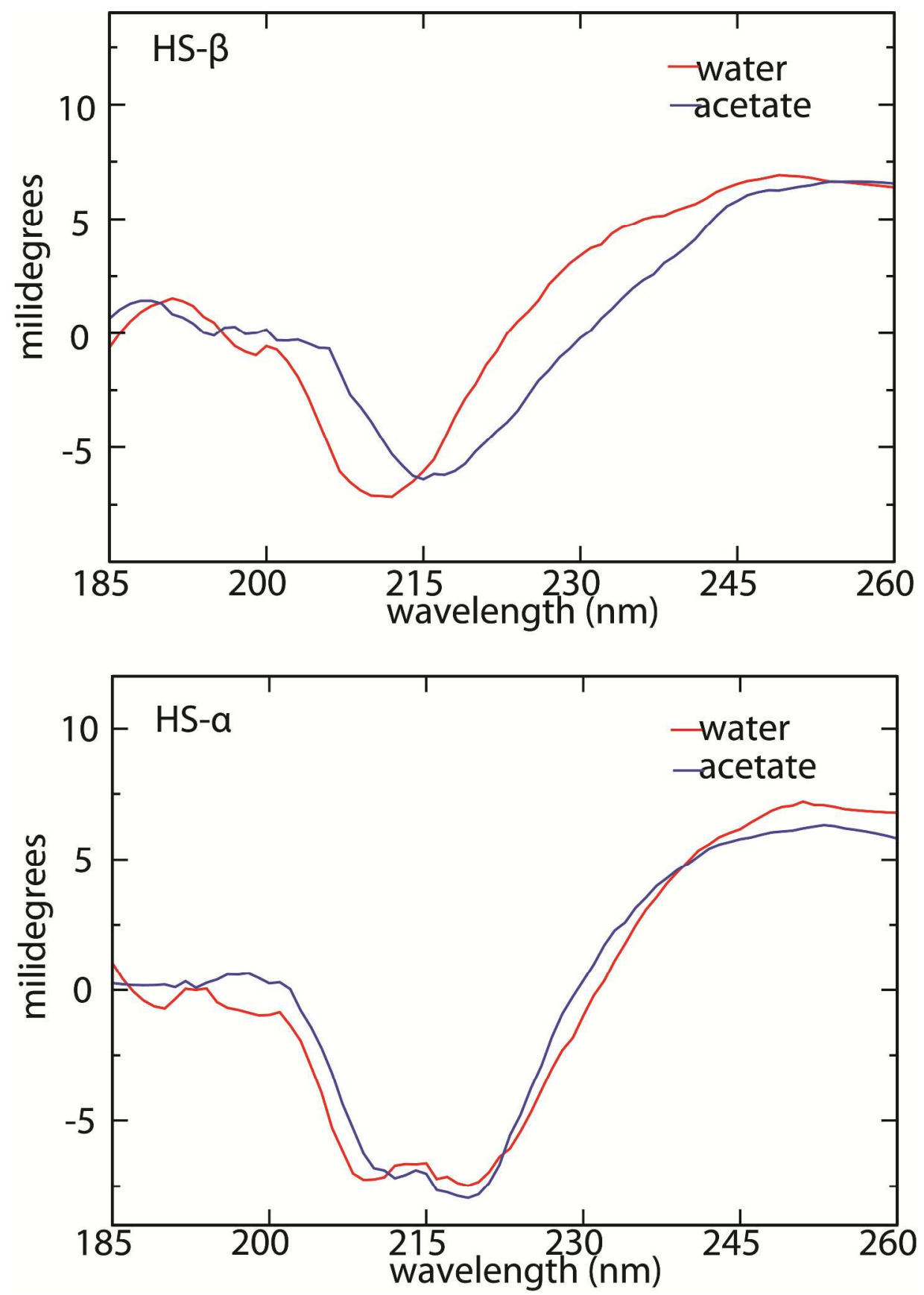

Figure S6. Far-UV spectra of HS- $\beta$ (top) and HS- $\alpha$ (bottom) in water and acetate buffer.

Table S1. The protein secondary structure analysis of the circular dichroism data. The CD spectra is fitted by KD2 method ${ }^{29}$ and the corresponding results of the abundance of the protein secondary structures are shown below. Dichroweb ${ }^{30-33}$ was used to analyze CD spectra. 


\begin{tabular}{|l|c|c|c|}
\hline Set/secondary structure & $\alpha$-helix & $\beta$-sheet & random coil \\
\hline HS- $\beta$ water & 0.24 & 0.4 & 0.36 \\
\hline HS- $\beta$ acetate & 0.21 & 0.54 & 0.25 \\
\hline HS- $\alpha$ water & 0.59 & 0.08 & 0.33 \\
\hline HS- $\alpha$ acetate & 0.57 & 0.12 & 0.31 \\
\hline
\end{tabular}

\section{S4. IM-MS}
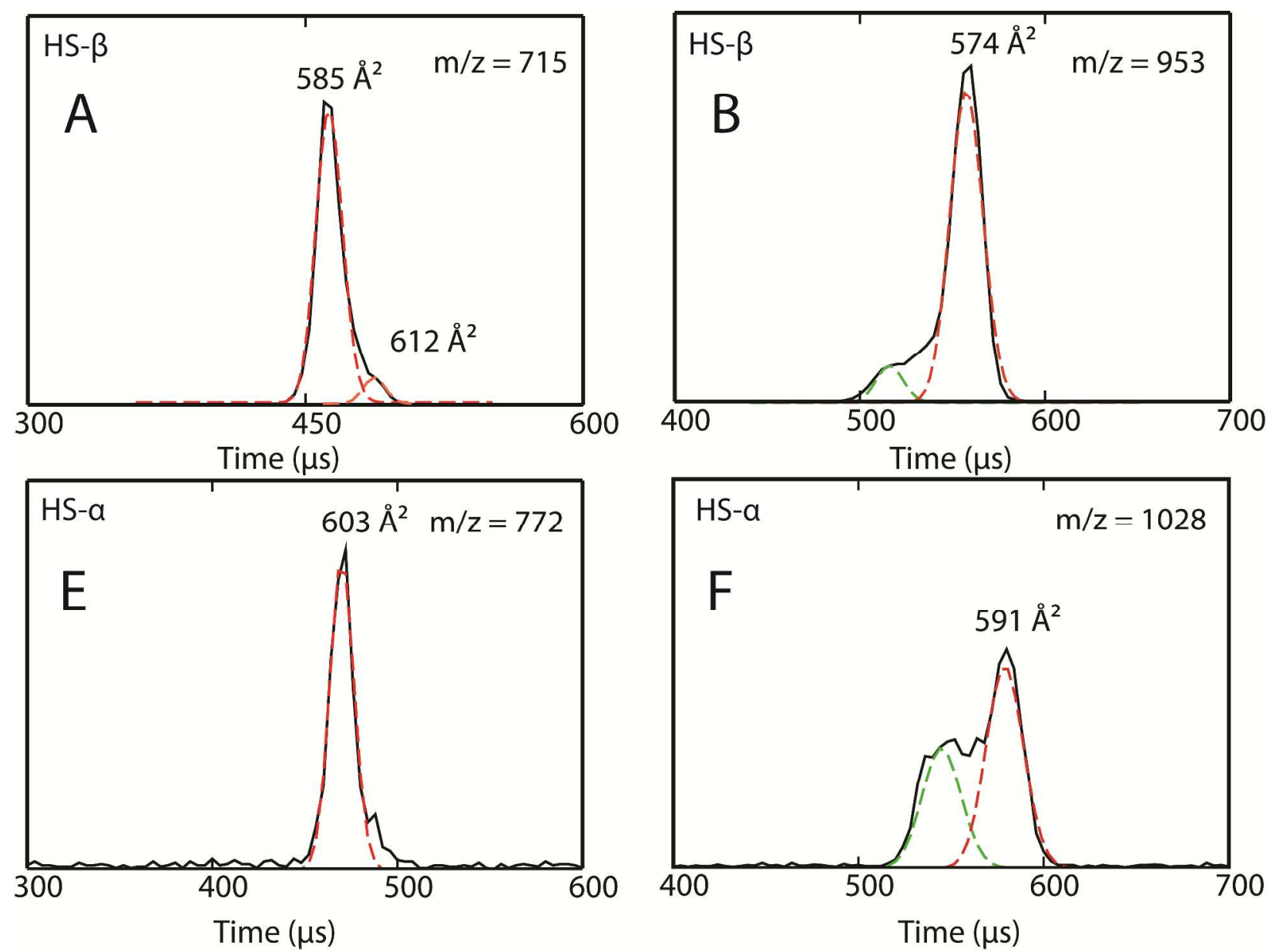

Figure S7. Representative ATDs for each $m / z$ value of HS- $\beta$ and HS- $\alpha$ in ammonium acetate buffer. The data were obtained by instrument 1 . The $\mathrm{m} / \mathrm{z}$ values correspond to charge states $z=$ 
+4 and +3 . ATD features corresponding to monomer are shown in red dash-line together with their cross sections.
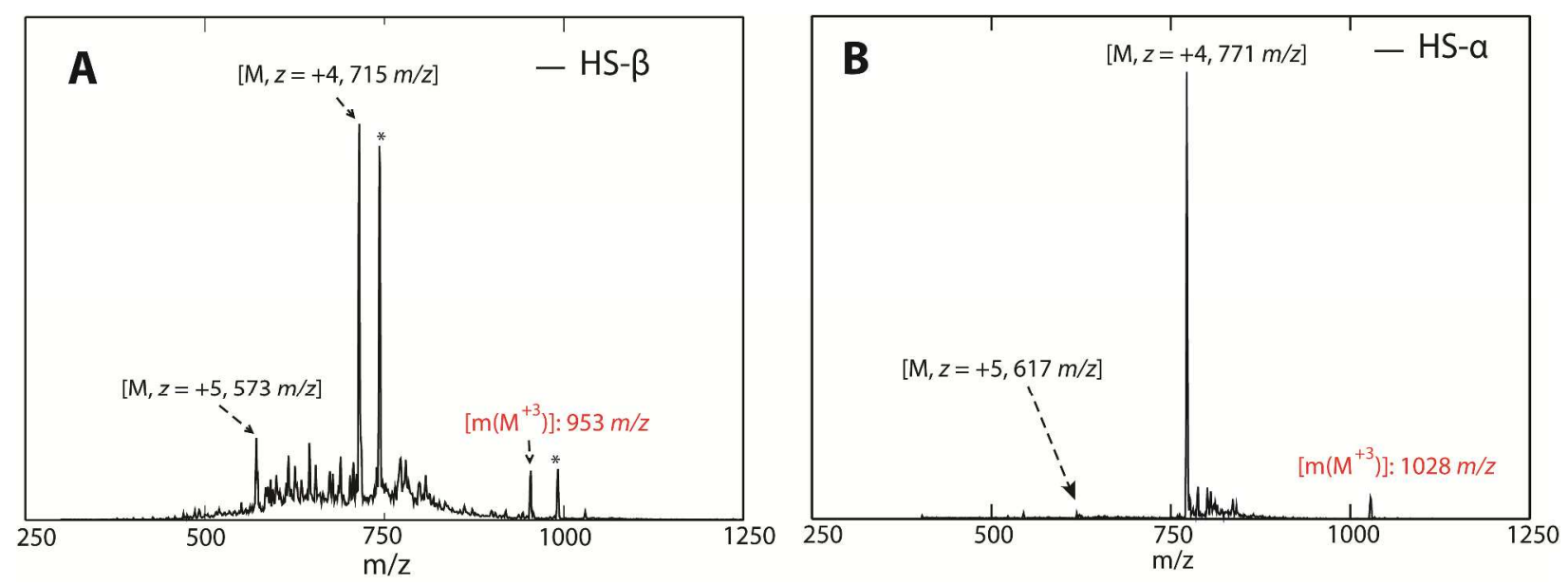

Figure S8. ESI-quad-mass spectra of (A) HS- $\beta$ and (B) HS- $\alpha$ in water obtained from instrument 2.
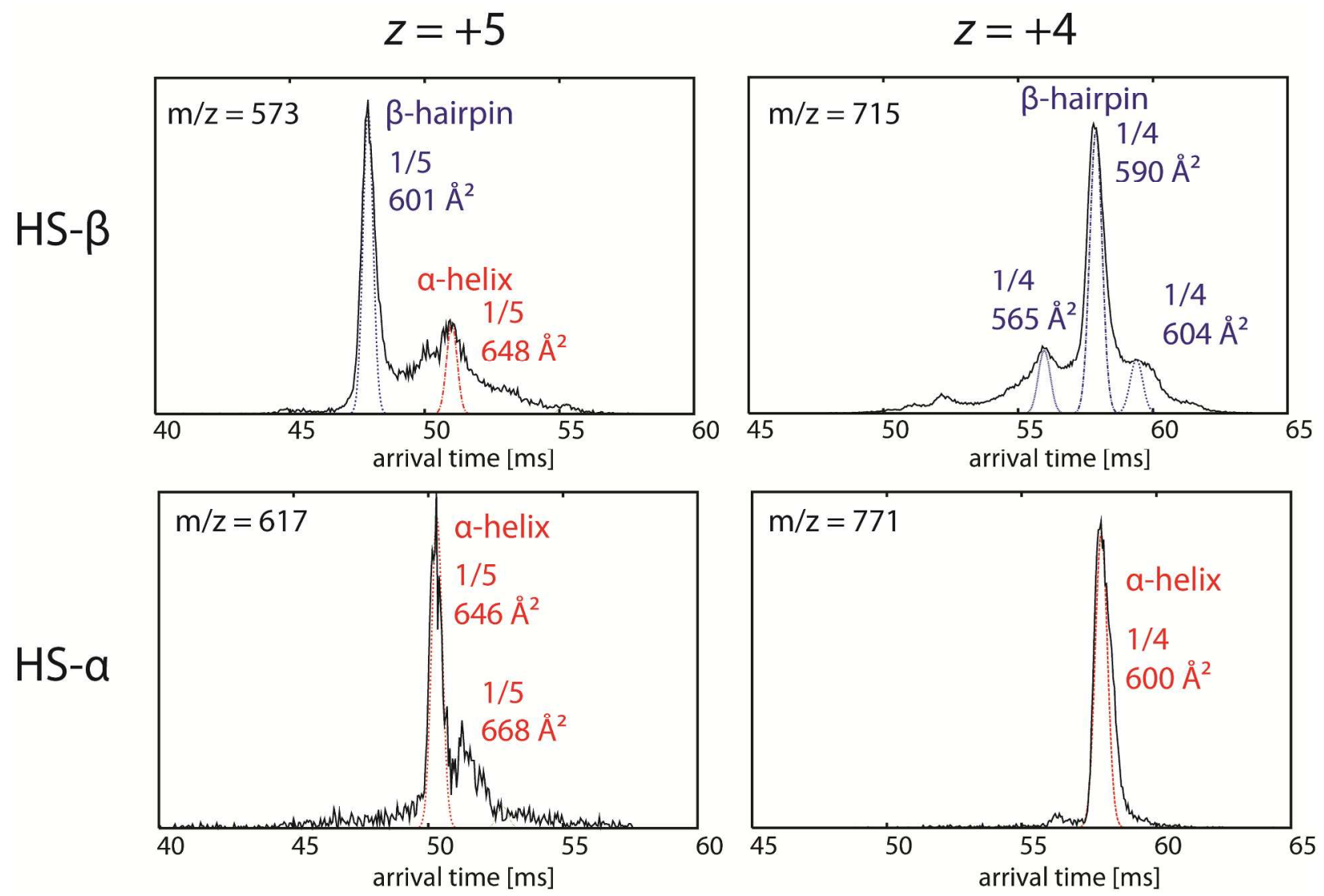
Figure S9. Representative ATDs for monomer peaks of HS- $\beta$ and HS- $\alpha$ in water. Data obtained using instrument 2 . The $m / z$ values correspond to charge states $z=+5,+4$. The cross sections of the monomers are shown.

\section{REFERENCES}

1. Sharma, A.; Sharma, I.; Kogkasuriyachai, D.; Kumar, N., Structure of a gametocyte protein essential for sexual development in plasmodium falciparum. Nat. Struc. Biol. 2003, 10, 197-203.

2. Knochel, T.; Ivens, A.; Hester, G.; Gonzalez, A.; Bauerle, R.; Wilmanns, M.; Kirschner, K.; Jansonius, J. N., The crystal structure of anthranilate synthase from sulfolobus solfataricus: Functional implications. Proc. Natl. Acad. Sci. U. S. A. 1999, 96, 9479-9484.

3. Humphrey, W.; Dalke, A.; Schulten, K., Vmd: Visual molecular dynamics. J. Mol. Graph. 1996, 14, 33-38.

4. Case, D. A.; Cheatham, T. E.; Darden, T.; Gohlke, H.; Luo, R.; Merz, K. M.; Onufriev, A.; Simmerling, C.; Wang, B.; Woods, R. J., The amber biomolecular simulation programs. $J$. Comp. Chem. 2005, 26, 1668-1688.

5. $\quad$ Case, D. A.; Darden, T. A.; T.E. Cheatham, I.; Simmerling, C. L.; Wang, J.; Duke, R. E.; Luo, R.; Merz, K. M.; Wang, B.; Pearlman, D. A.; Crowley, M.; Brozell, S.; Tsui, V.; Gohlke, H.; Mongan, J.; Hornak, V.; Cui, G.; Beroza, P.; Schafmeister, C.; Caldwell, J. W.; Ross, W. S.; Kollman, P. A. Amber 11, University of California: San Francisco, 2010.

6. Onufriev, A.; Bashford, D.; Case, D. A., Exploring protein native states and large-scale conformational changes with a modified generalized born model. Proteins 2004, 55, 383-394.

7. Ozkan, S. B.; Wu, G. A.; Chodera, J. D.; Dill, K. A., Protein folding by zipping and assembly. Proc. Natl. Acad. Sci. U. S. A. 2007, 104, 11987-11992.

8. Shell, M. S.; Ritterson, R.; Dill, K. A., A test on peptide stability of amber force fields with implicit solvation. J. Phys. Chem. B 2008, 112, 6878-6886.

9. Wu, C.; Murray, M. M.; Bernstein, S. L.; Condron, M. M.; Bitan, G.; Shea, J. E.; Bowers, M. T., The structure of a beta 42 c-terminal fragments probed by a combined experimental and theoretical study. J. Mol. Biol. 2009, 387, 492-501.

10. Loncharich, R. J.; Brooks, B. R.; Pastor, R. W., Langevin dynamics of peptides - the frictional dependence of isomerization rates of n-acetylalanyl-n'-methylamide. Biopolymers 1992, 32, 523-535.

11. Ryckaert, J. P.; Ciccotti, G.; Berendsen, H. J. C., Numerical integration of the cartesian equations of motion of a system with constraints: Molecular dynamics of n-alkanes. J. Comput. Phys. 1977, 23, 327-341.

12. Kabsch, W.; Sander, C., Dictionary of protein secondary structure: Pattern recognition of hydrogen-bonded and geometrical features. Biopolymers 1983, 22, 2577-2637.

13. Kumar, S.; Bouzida, D.; Swendsen, R. H.; Kollman, P. A.; Rosenberg, J. M., The weighted histogram analysis method for free-energy calculations on biomolecules .1. The method. J. Comp. Chem. 1992, 13, 1011-1021. 
14. Ferrenberg, A. M.; Swendsen, R. H., New monte-carlo technique for studying phasetransitions. Phys. Rev. Lett. 1988, 61, 2635-2638.

15. Ferrenberg, A. M.; Swendsen, R. H., Optimized monte-carlo data-analysis. Phys. Rev. Lett. 1989, 63, 1195-1198.

16. Feig, M.; Karanicolas, J.; Brooks, C. L., Mmtsb tool set: Enhanced sampling and multiscale modeling methods for applications in structural biology. J. Mol. Graph. Model. 2004, $22,377-395$.

17. Iavarone, A. T.; Williams, E. R., Mechanism of charging and supercharging molecules in electrospray ionization. J. Am. Chem. Soc. 2003, 125, 2319-2327.

18. Prell, J. S.; O'Brien, J. T.; Williams, E. R., Structural and electric field effects of ions in aqueous nanodrops. J. Am. Chem. Soc. 2011, 133, 4810-4818.

19. Iavarone, A. T.; Jurchen, J. C.; Williams, E. R., Effects of solvent on the maximum charge state and charge state distribution of protein ions produced by electrospray ionization. $J$. Am. Soc. Mass Spectrom. 2000, 11, 976-985.

20. Bernstein, S. L.; Wyttenbach, T.; Baumketner, A.; Shea, J.-E.; Bitan, G.; Teplow, D. B.; Bowers, M. T., Amyloid $\beta$-protein: Monomer structure and early aggregation states of a $\beta 42$ and its pro19 alloform. J. Am. Chem. Soc. 2005, 127, 2075-2084.

21. Bernstein, S. L.; Dupuis, N. F.; Lazo, N. D.; Wyttenbach, T.; Condron, M. M.; Bitan, G.; Teplow, D. B.; Shea, J.-E.; Ruotolo, B. T.; Robinson, C. V.; Bowers, M. T., Amyloid- $\beta$ protein oligomerization and the importance of tetramers and dodecamers in the aetiology of alzheimer's disease. Nat. Chem. 2009, 1, 326-331.

22. Chillier, X. F. D.; Monnier, A.; Bill, H.; Gulacar, F. O.; Buchs, A.; McLuckey, S. A.; Van Berkel, G. J., A mass spectrometry and optical spectroscopy investigation of gas-phase ion formation in electrospray. Rap. Comm. Mass Spectrom. 1996, 10, 299-304.

23. Sterling, H. J.; Prell, J. S.; Cassou, C. A.; Williams, E. R., Protein conformation and supercharging with dmso from aqueous solution. J. Am. Soc. Mass Spectrom. 2011, 22, 11781186.

24. Mason, E. A., Transport properties of ions in gases. 99 ed.; John Wiley \& Sons: 1988.

25. Hudgins, R. R.; Jarrold, M. F., Helix formation in unsolvated alanine-based peptides: Helical monomers and helical dimers. J. Am. Chem. Soc. 1999, 121, 3494-3501.

26. Gidden, J.; Ferzoco, A.; Baker, E. S.; Bowers, M. T., Duplex formation and the onset of helicity in poly $\mathrm{d}(\mathrm{cg})$ n oligonucleotides in a solvent-free environment. J. Am. Chem. Soc. 2004, 126, 15132-15140.

27. Humphrey, W.; Dalke, A.; Schulten, K., VMD: Visual molecular dynamics. J. Mol. Graph. Model. 1996, 14, 33-38.

28. Woody, R. W.; Koslowski, A., Recent developments in the electronic spectroscopy of amides and alpha-helical polypeptides. Biophys. Chem. 2002, 101, 535-551.

29. Andrade, M. A.; Chacon, P.; Merelo, J. J.; Moran, F., Evaluation of secondary structure of proteins from uv circular-dichroism spectra using an unsupervised learning neural-network. Protein Engineering 1993, 6, 383-390.

30. Whitmore, L.; Wallace, B. A., Dichroweb, an online server for protein secondary structure analyses from circular dichroism spectroscopic data. Nucleic Acids Res. 2004, 32, W668-W673.

31. Lobley, A.; Wallace, B. A., Dichroweb: A website for the analysis of protein secondary structure from circular dichroism spectra. Biophysical Journal 2001, 80, 373a-373a. 
32. Lobley, A.; Whitmore, L.; Wallace, B. A., Dichroweb: An interactive website for the analysis of protein secondary structure from circular dichroism spectra. Bioinformatics 2002, 18, 211-212.

33. Lees, J. G.; Miles, A. J.; Wien, F.; Wallace, B. A., A reference database for circular dichroism spectroscopy covering fold and secondary structure space. Bioinformatics 2006, 22, 1955-1962. 